J. Clin. Chem. Clin. Biochem.

Vol. 16, 1978, pp. 451-458

\title{
Ein Routineverfahren zur Untersuchung der Sauerstoffaffinität des Hämoglobins
}

\author{
Von O. Müller-Plathe \\ Zentrallaboratorium des Allgemeinen Krankenhauses Altona, Hamburg
}

(Eingegangen am 30. Dezember 1977/13. März 1978)

Zusammenfassung: Es wird eine Methode zur Messung des Halbsättigungsdrucks ( $\mathrm{p}_{50}$ ) und der ,standardisierten $\mathrm{O}_{2}$-Sättigung" als Maß für die Sauerstoffaffinität des Hämoglobins beschrieben. Für die Bestimmung ist außer einer normalen Blutgasanalyse lediglich eine Messung der $\mathrm{O}_{2}$-Sättigung nach Äquilibrierung des Blutes bei $\mathrm{pO}_{2}=31 \mathrm{~mm}$ $\mathrm{Hg}(4,12 \mathrm{kPa})$ erforderlich. Die Ablesung der Ergebnisse erfolgt aus einem speziell für diese Bestimmung konstruierten Nomogramm. Einsatzmöglichkeiten und Grenzen der Methode werden besprochen.

\section{A routine method for the investigation of the oxygen affinity of haemoglobin}

Summary: A method is described for the measurement of the half saturation pressure $\left(\mathrm{p}_{50}\right)$ and the "standardised $\mathrm{O}_{2}$-saturation", as an index of the oxygen affinity of haemoglobin. In addition to the normal blood gas analysis, the only other parameter required is the $\mathrm{O}_{2}$-saturation after equilibration of the blood at $\mathrm{pO}_{2}=31 \mathrm{~mm} \mathrm{Hg}(4.72 \mathrm{kPa})$. The results are read from a specially prepared nomogram. Potential uses and limitations of the method are discussed.

\section{Einfithrung}

Außer Atmung und Kreislauf beeinflußt vor allem die $\mathrm{O}_{2}$-Transportkapazität des Blutes die Sauerstoffversorgung des Organismus. Die $\mathrm{O}_{2}$-Transportkapazität wird in erster Linie von der Konzentration, aber auch von der $\mathrm{O}_{2}$-Affinität des Hämoglobins bestimmt. Die $\mathrm{Hb}-$ $\mathrm{O}_{2}$-Affinität wird jedoch im allgemeinen nicht untersucht, da die erforderlichen Maßnahmen - Aufnahme einer Sauerstoffbindungskurve oder Bestimmung des Halbsättigungsdrucks - als methodisch aufwendig gelten. Da die Veränderungen der $\mathrm{O}_{2}$-Affunität vor allem in der Intensivimedizin zunehmende Beachtung finden, wurde hier ein einfaches und apparativ wenig aufwendiges Verfahren zur quạntitativen Erfạssung der $\mathrm{Hb}^{-\mathrm{O}_{2}-}$ Affinität entwickelt.

\section{Material und Methoden}

\section{Prinzip}

Heparinisiertes Vollblut wird mit einem Gasgemisch von etwa $0,044 \mathrm{l} / 1 \mathrm{O}_{2}$, etwa $0,056 \mathrm{l} / 1 \mathrm{CO}_{2}$ und etwa $0,90 \mathrm{l} / 1 \mathrm{~N}_{2}$ bei $37^{\circ} \mathrm{C}$ äquilibriert. $\mathrm{s}_{2}$ der äquilibrierten Bluttprobe wird oxymetrisch gemessen. Die Werte für den nomographisch (auf $\mathrm{pH}=7,40$ und Basenabweichung $=0 \mathrm{mmol} / \mathrm{l}$ ) korrigierten $\mathrm{pO}_{2}$ des Äquilibriergases und $\mathrm{sO}_{2}$ werden in ein Nomogramm (Abb. 1) eingetragen. Dieses gestattet die Ablesung der korrigierten Werte für pso. Der aktuelle Wert für pso kann durch Berücksichtigung des aktuellen pH und der Basenabweichung errechnet werden. Bei dem geschilderten Verfahren wird voraus- gesetzt, daß die Untersuchung im Zusammenhang mit einer Blutgasanalyse erfolgt und daß die aktuellen Werte für $\mathrm{pH}$ und Basenabweichung bekannt sind.

\section{Geräte}

1. Blutgasanalysen-Gerät mit einer kombinierten Meßanordnung für $\mathrm{pH}, \mathrm{pCO}_{2}$ und $\mathrm{pO}_{2}$. Für die vorliegende Untersuchung wurde der Blutgasanalysator Corning $165^{1}$ ) benutzt. In dem Gerät wird $\mathrm{pH}$ mittels einer kapillaren Glaselektrode, $\mathrm{pO}_{2}$ mit einer membranüberżogenen Platinelektrode nach Clark (1) und $\mathrm{pCO}_{2}$ mit einer membranüberzogenen Glaselektrode nach Severinghaus (2) gemessen.

2. Oxymeter, das die Messung der Sauerstoffsättigung auf photometrischem Wege mit genügender Sicherheit im Bereich von 0,40 bis 0,80 zuläßt. Für die eigenen Untersuchungen wurde das CO-Oximeter IL $182^{2}$ ) benutzt, das neben der Bestimmung der $\mathrm{O}_{2}$-Sättigung die gleichzeitige Messung von Gesamt-Hb und $\mathrm{HbCO}$ gestattet.

3. Tonometer, das die Äquilibrierung von $4 \mathrm{ml}$ Blut bei $37 \pm 0,1^{\circ} \mathrm{C}$ mit wasserdampfgesättigten Gasen gestattet. Für die vorliegende Untersuchung wurde das IL-Tonometer $237^{2}$ ) verwendet.

4. PVC-Schlauch, etwa $0,5 \mathrm{~mm}$ Innendurchmesser.

1) Corning Medical, Medfield, Massachusetts 02052, USA. In der Bundesrepublik Deutschland: Fa. IMA Analysengeräte, 6300 Gießen, Postfach 5708.

2) Instrumentation Laboratory Inc., 113 Hartwell Avenue, Lexington, Massachusetts 02173, USA. - In der Bundesrepublik Deutschland: JL Boskamp GmbH., Kleinstraße 14, 5303 Hersel. 


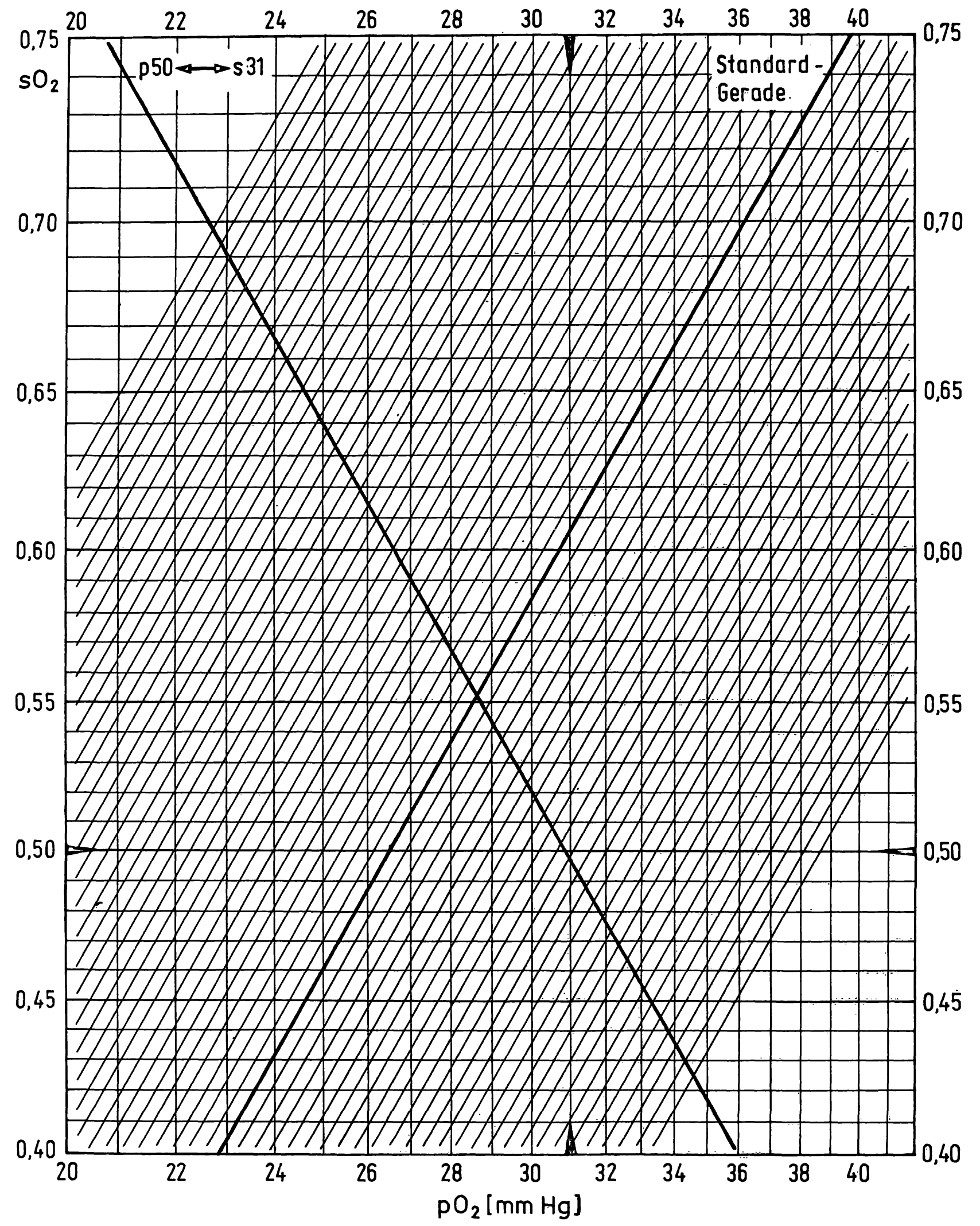

Abb. 1. Nomogramm zur Untersuchung der $\mathrm{O}_{2}$-Affinität.

Reagenzien

Für den Blutgasanalysator

Kalibrier-Pufferlösungen, Kalibriergase, Elektrolytlösungen für Elektroden; Reinigungs- und Spülflüssigkeiten entsprechend den Angaben des Geräteherstellers.
Für das CO-Oximeter

Null-Lösung und Reinigungsmittel entsprechend den Angaben des Geräteher stellers. 


\section{Äquilibriergas für das Tonometer}

Angestrebte Zusammensetzung $0,044 \mathrm{l} / 1 \mathrm{O}_{2}, 0,056 \mathrm{l} / 1 \mathrm{CO}_{2}$ und $0,90 \mathrm{l} / 1 \mathrm{~N}_{2}$ entsprechend $\mathrm{pO}_{2}=31 \mathrm{~mm} \mathrm{Hg}(4,12 \mathrm{kPa})$ und $\mathrm{pCO}_{2}=40 \mathrm{~mm} \mathrm{Hg}(5,32 \mathrm{kPa})$. Die Zusammensetzung dieses und aller bei methodischen Vergleichen verwendeten Gasgemische wurde mit dem Scholander-Apparat (3) im eigenen Laboratorium kontrolliert.

\section{Probenvorbereitung und -aufbewahrung}

Wie für blutgasanalytische $Z$ wecke üblich.

\section{Arbeitsweise}

Die Untersuchung der $\mathrm{O}_{2}$-Affinität wird zweckmäßig im Zusammenhang mit einer regulären Blutgasanalyse durchgeführt, da deren Ergebnisse für die Berechnung der aktuellen Werte von pso $_{50}$ erforderlich sind und da die Veränderungen der $\mathrm{O}_{2}$-Affinität ohne Kenntnis der Blutgasanalyse nur unvollständig zu interpretieren sind.

1. Für eine Doppelbestimmung werden $4 \mathrm{ml}$ Blut in das Äquilibriergefäß des IL-Tonometers eingefült. Gasstrom auf $300 \mathrm{ml} / \mathrm{min}$ einstellen. Motor anstellen. Äquilibrierdauer 20 min. - Vor Beginn der Analyse sicherstellen, daß die vorgeschriebene Wassermenge für die Gasbefeuchtung vorhanden.und die Temperatur von $37^{\circ} \mathrm{C}$ erreicht ist. Nach mehrstündigen Betriebspausen ist das Gerät 10 min lang mit Äquilibriergas vorzuspülen.

2. Während der Äquilibrierzeit Blutgasanalyse und Justierung des Oxymeters durchführen.

3. Nach Beendigung der Äquilibrierung Temperatur nochmals kontrollieren. Äquilibriergas aus dem Tonometer mittels einer Injektionsspritze etwa $30 \mathrm{~s}$ lang durch den Überführungsschlauch hindurchsaugen.

4. Blut über den so vorgespülten PVC-Schlauch durch zwei aufeinanderfolgende Transportzyklen unter Vermeidung von Luftkontakten in das $\mathrm{CO}-\mathrm{Oxymeter}$ einziehen. $\mathrm{sO}_{2}$ innerhalb $30 \mathrm{~s}$ ablesen und das Gerät mit Null-Lösung spülen (mindestens drei Zyklen). Die Nulleinstellungen sollen höchstens um $\pm 0,2$ abweichen. - Doppelbestimmung erforderlich.

\section{Berechnung des Halbsättigungsd rucks ( $\mathrm{p}_{50}$ )}

1. Auf dem Leiternomogramm von Siggaard-Andersen (Abb. 2) werden $\mathrm{pH}, \mathrm{pCO}_{2}, \mathrm{Hb}$ und Basenabweichung der aktuellen Blutgasanalyse eingetragen. Durch Drehung der sich hierbei ergebenden Geraden um den BA $^{3}$ )-Punkt wird das dem $\mathrm{pCO}_{2}$ des Äquilibriergases entsprechende $\mathrm{pH}$ aufgesucht.

2. Korrekturfaktoren $\mathrm{F}_{\mathrm{pH}}$ und $\mathrm{F}_{\mathrm{BA}}$ für $\mathrm{pO}_{2}$ mit Hilfe des Kelman-Nunn-Nomogramms (Abb. 3) autsuchen und den $\mathrm{pO}_{2}$ des Äquilibriergases mit diesen Faktoren multiplizieren.

3. Den Punkt für diesen korrigierten $\mathrm{pO}_{2}$ (Abszisse) und für $\mathrm{SO}_{2}$ (Ordinate) im Affinitäts-Nomogramm (Abb. 1) markieren.

4. Durch diesen Punkt eine Parallele zur eingezeichneten Standard:-Geraden ziehen.

5. Am Schnittpunkt der Parallelen mit der Horizontallinie $\mathrm{SO}_{2}=0,50 \mathrm{kann} \mathrm{p}_{50}$ (korr) abgelesen werden.

6. Korrekturfaktor $F_{\mathrm{pH}}$ für die aktuelle Blutgasanalyse im Kelman-Nunn-Nomogramm (Abb. 3) aufsuchen und pso(korr) durch $F_{\text {pH }}$ und den schon bekanninten $F_{B A}$ dividieren. Auf diese Weise erhält man pso(akt).

Wenn die genaue Zusammensetzung des Äquilibriergases nicht bekannt ist oder wenn man sịch einer verläßlichen Überführung der Proben in die Meßgeräte nicht sicher sein kann, muß $\mathrm{pO}_{2}$ in der äquilibrierten Blutprobe bestimmt und fụr weitere Berechnungen zugrundegelegt werden.
Berechnung der ,standardisierten $\mathrm{O}_{2}$-Sättigung“" (stand- $\mathrm{SO}_{2}$ )

Als weiteren möglichen Ausdruck für die $\mathrm{Hb}-\mathrm{O}_{2}$-Affinität, in der Diskussion ausführlich besprochen, sehen wir eine durch folgende Bedingungen standardisierte Sauerstoffsättigung an:

$$
\begin{array}{ll}
\mathrm{pO}_{2} & =31 \mathrm{~mm} \mathrm{Hg}(4,12 \mathrm{kPa}) \\
\mathrm{T} & =37^{\circ} \mathrm{C} \\
\mathrm{pH} & =7,40 \\
\text { Basen- } & \\
\text { abweichung } & =0 \mathrm{mmol} / 1
\end{array}
$$

Sind alle vier Bedingungen crfüllt, wird der Index ,korr" zugesetzt. Der Bezug auf die aktuellen Werte des pH und der Basenabweichung wird durch den Zusatz ,akt" (= aktuell) gekennzeichnet.

Man erhält stand-sO $\mathrm{O}_{2}$ (korr), wenn man den Schnittpunkt der eingezeichneten Parallelen mit der Vertikallinie $\mathrm{pO}_{2}=$ $31 \mathrm{~mm} \mathrm{Hg}$ aufsucht. Zudem erlaubt die mit $\mathrm{p}_{50} \leftrightarrow \mathrm{s}_{31}$ bezeichnete Gerade die Auffindung des zu einem bestimmten $p_{50}$ (Abszisse) gehörenden stand- $\mathrm{SO}_{2}$ (Ordinate).

Ein Berechnungsbeispiel zur Ermittlung der aktuellen und korrigierten Werte für $\mathrm{p}_{50}$ und stand- $\mathrm{SO}_{2}$ findet sich als Anhang $\mathrm{zu}$ dieser Arbeit.

Das Nomogramm zur Untersuchung der $\mathrm{O}_{2}$-Affinität (Abb. 1)

Dem Nomogramm liegt die Hillsche Gleichung (4) zugrunde, die die Beziehung zwischen $\mathrm{pO}_{2}$ und der Sauerstoff sättigung des Hämoglobins über den Sättigungsbereich zwischen 0,10 und 0,95 linearisiert darzustellen gestattet.

Die Hillsche Gleichung lautet

$$
\log \frac{\mathrm{sO}_{2}}{100-\mathrm{sO}_{2}}=\log \mathrm{K}+\mathrm{n} \log \mathrm{pO}_{2}
$$

Auf der Abszisse ist $\mathrm{pO}_{2}$ im logarithmischen Maßstab aufgetragen. Der Einteilung der Ordinate liegt entsprechend der linken Seite in der obigen Gleichung der dekadische Logarithmus des Verhältnisses zwischen oxygeniertem und deoxygeniertem Hämoglobin zugrunde. Zur bequemeren Handhabung ist die Ordinate mit den zugehörigen $\mathrm{sO}_{2}$-Werten beschriftet.

In das so gebildete Koordinatensystem wurde die Standard- $\mathrm{O}_{2}$ Dissoziationskurve nach Severinghaus (5) für den Sättigungsbereich von 0,40 bis 0,75 als Gerade eingezeichnet. Die Steilheit dieser Geraden hängt von dem für n gewählten Wert ab. Bei Berücksichtigung aller von Severinghaus angegebenen Werte zwischen 0,15 und 0,93 ergibt sich $\log K=-3,8403$ und $n=2,7081$.

Für die Konstruktion der Geraden wurde der Wert 2,7 eingesetzt. Die Standard-Gerade verläuft durch den Punkt für die Halbsättigung mit den Koordinaten 26,5 mm Hg und 0,50 für die $\mathrm{O}_{2}$-Sättigung.

Zur Erleichterung der für die Ablesung erforderlichen Parallelverschiebung wurden Parallelen zur Standard-ODK ${ }^{4}$ ) eingezeichnet. - Die von links oben nach rechts unten verlaufende Gerade stellt die Beziehung $\mathrm{Zwischen} \mathrm{p}_{50}$ und stand- $\mathrm{sO}_{2}$ dar. Mit ihrer Hilfe kann für jeden gegebenen $p_{50}$ die zugehörige stand- $\mathrm{sO}_{2}$ abgelesen werden und umgekehrt.

\section{Ergebnisse}

\section{Qualitätskriterien}

Die Präzision in der Serie geht aus der Tabelle 1, oberer Teil, hervor. Die Präzision von Tag zu Tag ist aufgrund der Natur des Untersuchungsmaterials nicht exakt zu

\footnotetext{
3) $\mathbf{B A}=$ Basenabweichung

4) $\mathrm{ODK}=\mathrm{O}_{2}$-Dissoziationskurve
} 


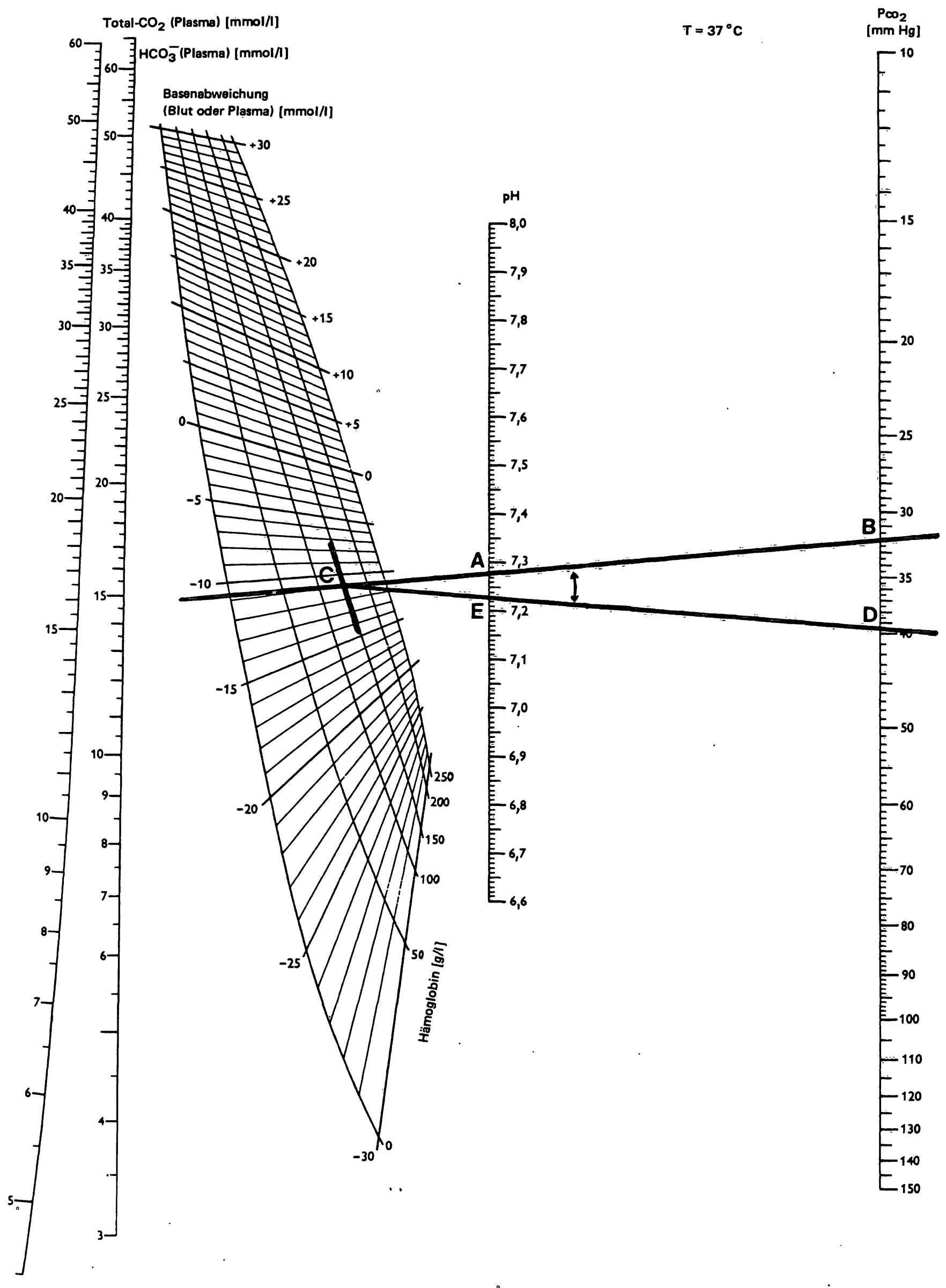

Abb. 2. Das Leiternomogramm nach Siggaard-Andersen (16) mit eingezeichnetem Berechnungsbeispiel (s. Anhang). Die Wiedergabe erfolgt mit der von Fa K. Hillerkus, Krefeld, bei Radiometer AS eingeholten Genehmigung. 


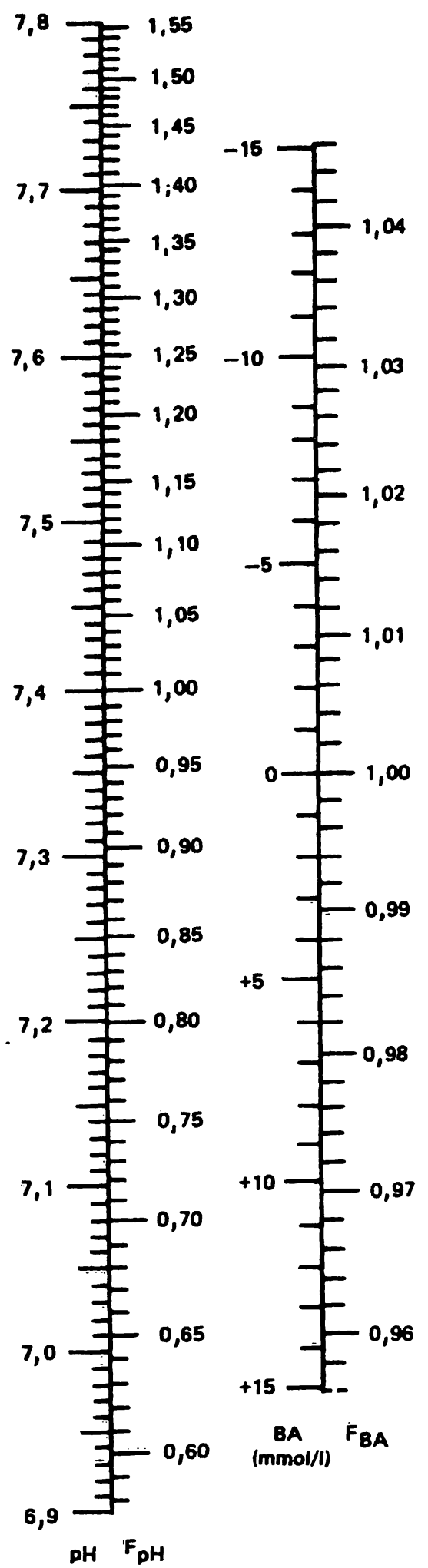

Abb. 3. Fàktoren für die $\mathrm{pO}_{2}$-Korrektur auf die Standardbedingungen $\mathrm{pH}$ 7,40 und Basenabweichung $0 \mathrm{mmol} / 1$. Diese Darstellung ist dem Nomogramm von Kelman und Nunn (17) entnommen.

bestimmen. Gewisse Anhaltspunkte für die Präzision über einen längeren Zeitraum ergeben sich aus Untersuchungen, die im Verlaụf vọ neụn Monaten bei einem Probanden durchgefuihrt wurden (Tab. 1, unterer Teil).
Tab. 1. Präzision der Bestimmung von $\mathrm{p}_{50}$ und stand-sO

\begin{tabular}{|c|c|c|c|}
\hline & Pso & & stand-sO $\mathrm{O}_{2}$ \\
\hline In der Serie & $\begin{array}{l}n \\
\bar{x} \\
s \\
s_{\text {rel }}\end{array}$ & $\begin{array}{l}12 \\
28,70 \mathrm{~mm} \mathrm{Hg}(3,82 \mathrm{kPa}) \\
0,28 \mathrm{~mm} \mathrm{Hg}(0,037 \mathrm{kPa}) \\
0,98 \%\end{array}$ & $\begin{array}{l}12 \\
0,588 \\
0,0064 \\
1,09 \%\end{array}$ \\
\hline $\begin{array}{l}\text { Bei wiederholten } \\
\text { Untersuchungen } \\
\text { an einem } \\
\text { Probanden }\end{array}$ & $\begin{array}{l}n \\
\bar{x} \\
s \\
\text { stel }_{\text {rel }}\end{array}$ & $\begin{array}{l}9 \\
27,03 \mathrm{~mm} \mathrm{Hg}(3,59 \mathrm{kPa}) \\
0,335 \mathrm{~mm} \mathrm{Hg}(0,045 \mathrm{kPa}) \\
1,24 \%\end{array}$ & $\begin{array}{l}9 \\
0,61 \\
0,0094 \\
1,55 \%\end{array}$ \\
\hline
\end{tabular}

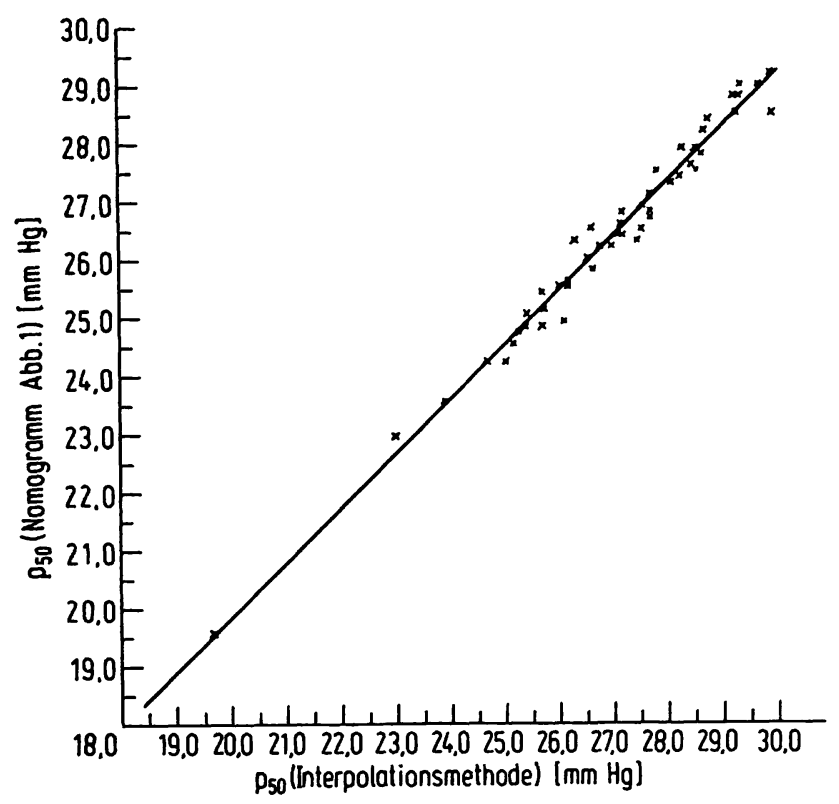

Abb. 4. Korrelation zwischen dem nomographisch (Ordinate) und dem mit der Interpolationsmethode (Abszisse) bestimmten Halbsättigungsdruck. $y=0,919+0,984 x ; n=49 ; r=0,989$

Die Richtigkeit wurde durch Vergleich mit der Interpolationsmethode (6) untersucht (Abb. 4). Hierbei ergab sich ein Korrelationskoeffizient von $r=0,989$.

Eine weitere Richtigkeitskontrolle wurde auf der Basis des Bohr-Effektes durchgeführt. In der jeweiligen Blutprobe wurde zunächst der aktuelle $\mathrm{p}_{50}$ nach dem hier geschilderten Verfahren bestimmt. Sodann wurden $\mathrm{pH}$ und Basenabweichung des Blutes durch Einrühren von $\mathrm{HCl}$ oder $\mathrm{NaOH}$ verändert. Der in diesen Proben gefundené aktuelle $p_{50}$ wurde verglichen mit demjenigen Wert, der sich nach dem Kelman-Nunn-Diagramm (Abb. 3) unter Berücksichtigung von $\mathrm{pH}$ und Basenabweichung als Sollwert ergibt. Die Ergebnisse sind in der Tabelle 2 aufgefuihrt. Unter diesen teilweise extremen Säure-Basen-Bedingungen betrug die prozentuale Abweichung 0,94-1,01.

\section{Normalbereiche}

Die Normalbereiche wurden durch Untersuchung von 81 gesunden Probanden im Alter von 16 bis 75 Jahren 
Tab. 2. Richtigkeitskontrollen der $\mathrm{p}_{50}$-Bestimmung mit Hilfe des Bohr-Effekts. Die Veränderungen der Basenabweichung und des $\mathrm{pH}$ wurden durch Zugabe von $1 \mathrm{~mol} / 1$ $\mathrm{HCl}$ bzw. $1 \mathrm{~mol} / 1 \mathrm{NaOH}$ bewirkt. Die erwarteten Werte wurden mit dem Kelman-Nunn-Nomogramm (17) ermittelt.

\begin{tabular}{|c|c|c|c|c|c|}
\hline \multirow[t]{2}{*}{ Nr. } & \multirow{2}{*}{$\begin{array}{l}\text { Basen- } \\
\text { abweichung } \\
\text { (mmol/l) }\end{array}$} & \multirow[t]{2}{*}{$\mathrm{pH}$} & \multicolumn{2}{|l|}{$\begin{array}{l}\text { pso(akt) } \\
\text { (mm Hg) }\end{array}$} & \multirow{2}{*}{$\begin{array}{l}\text { Uber- } \\
\text { ein- } \\
\text { stim- } \\
\text { mung }\end{array}$} \\
\hline & & & $\begin{array}{l}\text { ge- } \\
\text { messen }\end{array}$ & erwartet & \\
\hline 1. & $\begin{array}{l}+0,3 \\
-10,5 \\
+20,3\end{array}$ & $\begin{array}{l}7,39 \\
7,22 \\
7,63\end{array}$ & $\begin{array}{l}26,7 \\
30,8 \\
21,8\end{array}$ & $\begin{array}{l}- \\
31,1 \\
22,4\end{array}$ & $\begin{array}{l}\overline{0,99} \\
0,97\end{array}$ \\
\hline 2. & $\begin{array}{l}-1,6 \\
-16,1 \\
-21,5\end{array}$ & $\begin{array}{l}7,36 \\
7,11 \\
7,01\end{array}$ & $\begin{array}{l}26,1 \\
31,9 \\
34,9\end{array}$ & $\begin{array}{l}- \\
32,9 \\
36,2\end{array}$ & $\begin{array}{l}\overline{0,97} \\
0,96\end{array}$ \\
\hline 3. & $\begin{array}{r}2,5 \\
-16,9 \\
+12,0\end{array}$ & $\begin{array}{l}7,35 \\
7,11 \\
7,52\end{array}$ & $\begin{array}{l}25,7 \\
32,8 \\
22,2\end{array}$ & $\begin{array}{l}- \\
32,4 \\
22,3\end{array}$ & $\begin{array}{l}\overline{1}, 01 \\
1,00\end{array}$ \\
\hline 4. & $\begin{array}{l}-1,3 \\
-\quad 8,4 \\
+\quad 3,8\end{array}$ & $\begin{array}{l}7,36 \\
7,25 \\
7,41\end{array}$ & $\begin{array}{l}25,7 \\
26,6 \\
25,1\end{array}$ & $\begin{array}{l}- \\
28,2 \\
24,9\end{array}$ & $\begin{array}{l}\overline{0,94} \\
1,01\end{array}$ \\
\hline
\end{tabular}

ermittelt. Es handelt sich um 45 Männer (Durchschnittsalter 40,3 Jahre) und 36 Frauen (Durchschnittsalter 41,6 Jahre). Probanden, bei denen sich durch begleitende Untersuchungen Erkrankungen der Atem- und Kreislauforgane, der Leber oder Niere herausstellten, sowie Patienten mit Hämoglobinwerten unter $12 \mathrm{~g} / \mathrm{dl}$ wurden ausgeschlossen. Als Normalbereich ergab sich für $\mathrm{p}_{50} 25,1-28,3 \mathrm{~mm} \mathrm{Hg}(3,34-3,76 \mathrm{kPa})$ und für stand- $\mathrm{sO}_{2} \quad 0,561-0,639$.

Wie aus Tabelle 3 hervorgeht, besteht eine deutliche Geschlechtsabhängigkeit. Frauen haben eine geringere $\mathrm{O}_{2}$-Affinität als Männer. $\mathrm{p}_{50}$ liegt bei ihnen um $0,5 \mathrm{~mm} \mathrm{Hg}$ höher und stand-sO $\mathrm{O}_{2}$ um 0,019 tiefer als bei Männern. Beide Unterschiede sind statistisch signifikant $(p<0,001)$. Die Werte sind normal verteilt.

\section{Haltbarkeit des Materials}

Aufbewahrung bei $+4{ }^{\circ} \mathrm{C}$ in luftdicht verschlossenen Injektionsspritzen aus Glas verändert im Laufe eines Arbeitstages die Werte für $\mathrm{p}_{50}$ und stand- $\mathrm{SO}_{2}$ nicht.

\section{Störfaktoren}

Venöse Stauung führt nach unseren Erfahrungen zu einer leichten Zunahme von $\mathbf{p}_{\mathbf{5 0}}$. Blut sollte deshalb am besten durch Arterienpunktion, auf jeden Fall aber ohne venöse Stauung abgenommen werden.

\section{Diskussion}

Die vorstehend geschilderte Methỏde zur Bestimmung des Halbsättigungsdrucks und der standardisierten $\mathrm{O}_{2}$ Sättigung bei $\mathrm{pO}_{2}=31 \mathrm{~mm} \mathrm{Hg}(4,12 \mathrm{kPa})$ wurde in der Absicht entwickelt, mit begrenztem apparativen und zeitlichen Aufwạnd eine möglichst präzise Information über die Sauerstoffaffinität des Hämoglobins als Ergänzung zur Routine-Blutgasanalyse zu erhalten. Es hat sich gezeigt, daß beide Größen durch eine einzige Äquilibrierung mit guter Präzision und Richtigkeit bestimmt werden können. Voraussetzung hierfür ist die Verwendung des speżiell für diese Bestimmung von uns entwickelten Nomogramms (Abb. 1). Seine Koordinaten sind so gewählt, daß sich die $\mathrm{O}_{2}$-Bindung des Hämoglobins in Abhängigkeit von $\mathrm{pO}_{2}$ als Gerade darstellt im Gegensatz zum s-förmigen Verlauf der herkömmlichen $\mathrm{O}_{2}$-Bindungskurve. Wird die durch das Hillsche $\mathrm{n}$ gegebene Steilheit der Geraden als konstant angenommen, so ist der Verlauf der Geraden durch einen einzigen Äquilibrierpunkt zu bestimmen.

Der für das Nomogramm gewählte Wert $n=2,7$ stimmt mit neueren Literaturangaben $(7,8,9)$ und auch mit eigenen Untersuchungen gut überein. Er schwankt normalerweise nur in engen Grenzen.

Tab. 3. Normalbereiche für $\mathrm{p}_{50}$ und stand- $\mathrm{SO}_{2}$

\begin{tabular}{|c|c|c|c|c|c|}
\hline $\mathrm{n}$ & & & $\begin{array}{l}\text { Männer } \\
45\end{array}$ & $\begin{array}{l}\text { Frauen } \\
36\end{array}$ & $\begin{array}{l}\text { Alle } \\
81\end{array}$ \\
\hline p50 $_{50}$ & $\begin{array}{l}\bar{x} \\
s \\
s_{T e 1} \\
\bar{x} \pm 2 s\end{array}$ & $\begin{array}{l}(\mathrm{mm} \mathrm{Hg}) \\
(\mathrm{mm} \mathrm{Hg}) \\
(\%) \\
(\mathrm{mm} \mathrm{Hg})\end{array}$ & $\begin{array}{cc}26,4 & \\
0,67 & \\
2,54 & \\
25,1 & -27,7\end{array}$ & $\begin{array}{ll}27,1 & \\
0,74 & \\
2,74 & \\
25,6 & -28,6\end{array}$ & $\begin{array}{cc}26,7 & \\
0,80 & \\
3,00 & \\
25,1 & -28,3\end{array}$ \\
\hline P.50 & $\begin{array}{l}\bar{x} \\
s \\
s_{\text {rel }} \\
\bar{x} \pm 2 s\end{array}$ & $\begin{array}{l}(\mathrm{kPa}) \\
(\mathrm{kPa}) \\
(\%) \\
(\mathrm{kPa})\end{array}$ & $\begin{array}{l}3,51 \\
0,089 \\
2,54 \\
3,34-3,68\end{array}$ & $\begin{array}{l}3,60 \\
0,098 \\
2,74 \\
3,40-3,80\end{array}$ & $\begin{array}{l}3,55 \\
0,106 \\
3,00 \\
3,34-3,76\end{array}$ \\
\hline stand-s $\mathrm{O}_{2}$ & $\begin{array}{l}\bar{x} \\
s \\
S_{\text {Tel }} \\
\bar{x} \pm 2 s\end{array}$ & $\%$ & $\begin{array}{l}0,609 \\
0,016 \\
2,64 \\
0,577-0,641\end{array}$ & $\begin{array}{l}0,590 \\
0,018 \\
3,03 \\
0,554-0,626\end{array}$ & $\begin{array}{l}0,600 \\
0,019 \\
3,21 \\
0,561-0,639\end{array}$ \\
\hline
\end{tabular}


Schwankungen von $\mathrm{pH}, \mathrm{pCO}_{2}$, Glycerat-2, 3-biphosphat, MCHC und des Elektrolytmilieus, die in erster Linie durch eine Beeinflussung der Salzbrücken im Hb-Tetramer eine der Konformationen des Moleküls stabilisieren oder destabilisieren, bewirken bevorzugt eine Parallelverschiebung der Dissoziationslinie.

Veränderungen der Steilheit treten vorwiegend dann auf, wenn die kooperativen Eigenschaften der Ketten selbst verändert sind wie bei einigen Hämoglobinopathien. Beim Vorliegen einer Hämoglobinopathie können sich bei jeder „Einpunktmethode" zur $\mathrm{p}_{50}-\mathrm{Be}$ stimmung Fehler ergeben, die umso größer sind, je weiter der Äquilibrierpunkt vom Bereich der Halbsättigung entfernt ist. Schon aus diesen Gründen halten wir im Gegensatz zu Aberman et al (10) und Lichtman et al (8), die $\mathrm{pO}_{2}$ und $\mathrm{sO}_{2}$ aus peripherem Venenblut bestimmen und auf jegliche Äquilibrierung verzichten, die Äquilibrierung in Halbsättigungsnähe für dringend erforderlich.

Für die Wahl des Äquilibriergas- $\mathrm{pO}_{2}$ von $31 \mathrm{~mm} \mathrm{Hg}$ $(4,12 \mathrm{kPa})$ waren mehrere Gründe maßgebend:

1. Die zugehörige $\mathrm{sO}_{2}$ beträgt normalerweise 0,60 , liegt also in der Nähe der Halbsättigung, was für die Richtigkeit der $\mathrm{p}_{50}$-Bestimmung von Vorteil ist.

2. Methodische Gründe lassen es ratsam erscheinen, eine standardisierte $\mathrm{sO}_{2}$-Bestimmung mit vorangehender Äquilibrierung und Probenüberführung im oberen Bereich des steilen Anteils der Sauerstoffbindungskurve vorzunehmen. Einerseits kommt für die deutliche Erkennung der Verschiebung nur der steile Bereich in Betracht. Andererseits haben Untersuchungen im eigenen Labor ergeben, daß der methodische Variationskoeffizient der $\mathrm{sO}_{2}$-Bestimmung mit Abnahme von $\mathrm{sO}_{2}$ beträchtlich zunimmt (11).

3. Mit diesem Verfahren soll nicht nur $p_{50}$ bestimmt werden können, sondern auch die Standard-sO $\mathrm{O}_{2}$, also diejenige Sättigung des Hämoglobins bei $\mathrm{pO}_{2}=$ $31 \mathrm{~mm} \mathrm{Hg}(4,12 \mathrm{kPa})$ (weitere Standardisierungsbedingungen s. Abschnitt Berechnung). Der Normalwert des $\mathrm{pO}_{2}$ im Sinus sagittalis beträgt $34 \mathrm{~mm} \mathrm{Hg}$ (4,52 kPa) (12). Erste Beeinträchtigungen der Hirntätigkeit (EEG-Veränderungen und Verschlechterung psychologischer Testleistungen) findet man schon beim Abșinken auf 27-29 $\mathrm{mm} \mathrm{Hg}(3,59-3,86 \mathrm{kPa})$ (13). Der $\mathrm{O}_{2}$-Druck von $31 \mathrm{~mm} \mathrm{Hg}(4,12 \mathrm{kPa})$ markiert somit den pathophysiologisch bedeutsamen Grenzbereich, bei dessen Unterschreiten erste Zeichen cerebraler Funktionsbeeinträchtigung eintreten.

\section{Klinische Bedeutung}

$\mathrm{pH}, \mathrm{pCO}_{2}$, Temperatur, $\mathrm{COHb}$-Gehalt und die Glycerat2, 3-bisphosphat-Konzentration in den Erythrocyten sind die wichtigsten Regulatoren der $\mathrm{H}_{\mathrm{b}}-\mathrm{O}_{2}$-Affinität. Dementsprechend hat die Untersuchung der Affinitätsparameter große Bedeutung für die Intensivmedizin. Hypoxie, Acịdose und Alkalose, Anämien, Schock und
CO-Vergiftung führen bekanntlich zu teilweise erheblichen Verschiebungen der $\mathrm{Hb}-\mathrm{O}_{2}$-Affinität. Wie die Massentransfusion (14) führt auch die plötzliche Korrektur oder gar Überkorrektur einer Acidose (15) vorübergehend zu einer starken Zunahme der $\mathrm{O}_{2}$-Affinität und damit zur Verschlechterung der Sauerstoffversorgung der Gewebe.

Die Kenntnis der Affinitäts-Parameter setzt den Kliniker in die Lage, derartige Risiken zu erkennen und zu vermeiden. Die wichtigsten Ursachen für Veränderungen der $\mathrm{Hb}-\mathrm{O}_{2}$-Affinität sind in Tabelle 4 aufgeführt.

Die Kenntnis von stand- $\mathrm{sO}_{2}$ und der Hämoglobinkonzentration ermöglicht auf einfache Weise die Berechnung der $\mathrm{O}_{2}$-Transportkapazität des Blutes. Es kann berechnet werden, wieviel $\mathrm{ml} \mathrm{O}_{2}$ von 11 des betreffenden Blutes abgegeben werden, wenn eine $\mathrm{O}_{2}$-Verminderung vom aktuellen Wert bis auf den pathophysiologisch bedeutsamen Bereich um $31 \mathrm{~mm} \mathrm{Hg}(4,12 \mathrm{kPa})$ eintritt. Die unter diesen Umständen abgegebene $\mathrm{O}_{2}$ Menge ist im Folgenden mit $\mathrm{DO}_{2(31)}$ bezeichnet. Sie beträgt normalerweise $60-80 \mathrm{ml} / \mathrm{l}$ und läßt sich wie folgt berechnen:

$$
\mathrm{DO}_{2(31)}=\Delta \mathrm{sO}_{2} \times \mathrm{Hb}(\mathrm{g} / \mathrm{l}) \times 1,39
$$

Beispiel: $\mathrm{Hb}=125 \mathrm{~g} / \mathrm{l} ; \mathrm{pH}_{(\mathrm{akt})}=7,50$

$$
\begin{aligned}
& \mathrm{sO}_{2 \text { (akt) } \quad 0,966} \\
& \begin{array}{cc}
\text { stand- } \mathrm{sO}_{2 \text { (akt) }} & 0,672 \\
\hline \Delta \mathrm{sO}_{2 \text { (akt) }} & 0,294
\end{array}
\end{aligned}
$$

Aktueller Wert für $\mathrm{DO}_{2(31)}: 51,1 \mathrm{ml} / 1$

\begin{tabular}{cc}
$\mathrm{sO}_{2 \text { (korr) }}$ & 0,956 \\
stand-sO & 0,602 \\
\hline$\Delta \mathrm{sO}_{2(\text { korr) }}$ & 0,354 \\
$\mathrm{DO}_{2(31)}$ bei pH 7,40: & $61,5 \mathrm{ml} / 1$
\end{tabular}

Tab. 4. Ursachen für Veränderungen der $\mathrm{O}_{2}$-Affinität ( $\mathrm{p}_{50}$ und

\begin{tabular}{|c|c|}
\hline Abnahme der $\mathrm{O}_{2}$-Affinität & Zunahme der $\mathrm{O}_{2}$-Affinität \\
\hline $\begin{array}{ll}\text { Pso(korr) } & \text { erhöht } \\
\text { stand-sO }_{2} \text { (korr) } & \text { vermindert }\end{array}$ & $\begin{array}{ll}\text { P5o(korr) } & \text { vermindert } \\
\text { stand-sO }_{2(\text { korr) }} & \text { erhöht }\end{array}$ \\
\hline $\begin{array}{l}\text { Hypoxie } \\
\text { Höhenatmung } \\
\text { Herzinsuffizienz } \\
\text { Rechts-Links-Shunt } \\
\text { respiratorische Insuffizienz } \\
\text { (ohne Acidose) }\end{array}$ & $\begin{array}{l}\text { Acidose } \\
\text { Chronische respiratorische } \\
\text { Acidose } \\
\text { diabetische Acidose } \\
\text { renale Acidose (Urämie) } \\
\text { Lactatacidose, z. B. durch } \\
\text { Schock, Biguanide, } \\
\text { bakterielle Toxine }\end{array}$ \\
\hline $\begin{array}{l}\text { Anämie } \\
\text { Lebercirrhose } \\
\text { Chronische Alkalose } \\
\text { Hyperphosphatämie }\end{array}$ & $\begin{array}{l}\text { Massivtransfusion } \\
\text { CO-Vergiftung } \\
\text { Methämoglobinämie } \\
\text { Hypophosphatämie } \\
\text { Neugeborene (HbF) }\end{array}$ \\
\hline $\begin{array}{l}\text { Schwangerschaft } \\
\text { Hämoglobinopathien } \\
\text { Hyperthyreose } \\
\text { Schilddrüsenhormone }\end{array}$ & $\begin{array}{l}\text { Hämoglobinopathien } \\
\text { Angeb. Hexokinasemangel } \\
\text { Glycerat-2, 3-bisphosphat- } \\
\text { mutase-Mangel }\end{array}$ \\
\hline
\end{tabular}
stand- $\mathrm{SO}_{2}$ korrigiert für $\mathrm{pH} \mathrm{7,40} \mathrm{und} \mathrm{Basenabweichung}$ $0 \mathrm{mmol} / \mathrm{l}$ ).

Angeb. Pyruvatkinasemangel 
Die von $11 \mathrm{Blut}$ bei $\mathrm{pO}_{2}=31 \mathrm{~mm} \mathrm{Hg}$ abgegebene $\mathrm{O}_{2}$-Menge ist folglich unter den aktuellen Bedingungen des Patienten um $10,4 \mathrm{ml}$ entsprechend $17 \%$ reduziert. Dieses Abgabedefizit müßte im vorliegenden Falle durch eine Erhöhung der Organperfusion und wahrscheinlich auch des Herzzeitvolumens vom Organismus aufgefangen werden. Die damit verbundene Belastung kann durch eine exakte $\mathrm{pH}$-Einstellung vermieden werden. Diese einfache Berechnung ist, ausgehend vom Halbsättigungsdruck, unmittelbar nicht möglich.

\section{Anhang}

Beispiel zur Berechnung von $\mathrm{p}_{50}$ und stand- $\mathrm{sO}_{2}$ Meßergebnisse der aktuellen Blutgasanalyse:

$$
\begin{array}{ll}
\mathrm{pH} & 7,28(\mathrm{~A}) \\
\mathrm{pCO} & 32,0 \mathrm{~mm} \mathrm{Hg}(\mathrm{B}) \\
\text { Hämoglobin } & 150 \mathrm{~g} / \mathrm{l}
\end{array}
$$

Durch Eintragen auf dem Leiternomogramm (Abb. 2) entsteht die Gerade AB. Deren Verlängerung trifft die $\mathrm{Hb}$-Linie für $150 \mathrm{~g} / 1$ bei C. C markiert die Basenabweichung mit - 11,0 mmol/l. Die Gerade CB wird durch Drehung um C auf den $\mathrm{pCO}_{2}$ den Äquilibriergases $(39,5 \mathrm{~mm} \mathrm{Hg}$, Punkt D) eingestellt. An ihrem Schnittpunkt mit der pH-Skala wird das $\mathrm{pH}$ der Blutprobe mit 7,23 (Punkt E) abgelesen.

$\mathrm{pO}_{2}$ im Äquilibiergas betrage $31,0 \mathrm{~mm} \mathrm{Hg}$. Aus dem Nomogramm Abbildung 3 sind die Korrekturfaktoren 0,83 für

\section{Literatur}

1. Clark, L. C. (1956), Trans. Soc. Art. Int. Organs 2, 41.

2. Severinghaus, J. W. \& Bradley, A. F. (1958), J. Appl. Physiol. 13, 515-520.

3. Scholander, P. F. (1947), J. Biol. Chem. 167, 235-250.

4. Hill, A. W. (1910), J. Physiol. (London) 40, IV-VII.

5. Severinghaus, J. W. (1966), J. Appl. Physiol. 21, 11081116.

6. Fallon, K. D. (1970), The oxyhemoglobin dissociation curve: the significance of $\mathbf{p}_{50}$. Instrumentation Laboratory, Lexington, U.S.A.

7. Edwards, M. J., Novy, M. J., Walters, C. L. \& Metcalfe, J. (1968), J. Clin. Invest. 47, 1851-1857.

8. Lichtman, M. A., Murphy, M. S. \& Adamson, J. W. (1976), Ann. Intern. Med. 84, 517-520.

9. Siggaard-Andersen, O. (1974), The acid-base status of the blood. Munksgaard, Kopenhagen.

10. Aberman, A., Cavanilles, J. M., Weil, M. H. \& Shubin, H. (1975), J. Appl. Physiol. 38, 171-176.
pH 7,23 und 1,034 für BA $=-11,0 \mathrm{mmol} / 1 \mathrm{zu}$ entnehmen. Der $\mathrm{pO}_{2}$ des Åquilibriergases ist mit diesen-Faktoren zu multiplizieren:

$31,0(\mathrm{~mm} \mathrm{Hg}) \cdot 0,83 \cdot 1,034=26,6 \mathrm{~mm} \mathrm{Hg}$.

Meßergebnis für $\mathrm{sO}_{2}$ nach der Äquilibrierung: 0,605.

Im Affinitätsnomogramm Abbildung 1 den Punkt mit den Koordinaten $\mathrm{pO}_{2}=26,6 \mathrm{~mm} \mathrm{Hg}$ und $\mathrm{sO}_{2}=0,605$ aufsuchen. Die zu der Standardgeraden durch diesen Punkt gezogene Parallele schneidet die Horizontallinie für $\mathrm{sO}_{2}=0,5$ bei $22,8 \mathrm{~mm} \mathrm{Hg}$ (pso(korr)) und die Vertikallinie für $\mathrm{pO}_{2}=$ $31 \mathrm{~mm} \mathrm{Hg}$ bei sO 20,698 (stand $=\mathrm{s}_{2}$ (korr)).

Die Division von p $_{5}$ (korr) durch die Korrekturfaktoren für die Basenabweichung $(1,034)$ und den aktuellen pH:Wert $(0,88)$ ergibt pso(akt) mit 25,1 mm Hg. Für diesen Wert ergibt sich auf $\mathrm{p}_{50} \leftrightarrow \mathrm{s}_{31}$ ein stand-sO $\mathrm{O}_{2}$ (akt) von 0,64 .

\section{Anmerkung}

Für Routinezwecke kann der Einfluß der bei der Deoxygenation arterieller Blutproben auf $\mathrm{sO}_{2} \approx \dot{0}, 60$ freigesetzten Basen vernachlässigt werden. Hierdurch wird $\mathrm{p}_{50}(\mathrm{korr})$ um etwa 0,2 . mm Hg zu niedrig bestimmt. - Bei der Deoxygenation von $1 \mathrm{~g} \mathrm{Hb}$ werden $0,019 \mathrm{mmol}$ Base freigesetzt. Für genaue Messungen kann BA korrigiert weriden nach der Formel:

$$
\Delta \mathrm{BA}=\mathrm{Hb}(\mathrm{g} / \mathrm{l}) \times \Delta \mathrm{sO}_{2} \times 0.019
$$

Bei der nomographischen Bestimmung des pH in der äquilibrierten Probe (s. Berechnung des Halbsättigungsdrucks, Punkt 1) müßte mañ dann von der um $\triangle \mathrm{BA}$ korrigièrten Basenabweịchung ausgehen.
11. Bela, U. (1975), Untersuchungen zur Bestimmung der Sauerstoffsättigung und der Sauerstoffaffinität des Hämoglobins. Dissertation, Hamburg.

12. Opitz, E. \& Schneider, M. (1950), Ergeb. Physiol. 46, 126-260.

13. Ernsting, J. (1966) in: Oxygen measurements in blood and tissue. Payne, J. P. \& Hill, D. W., eds.) Chur chill, London.

14. Junger, H., (1977), Bluttransfusion und Empfängerorganismus. Thieme, Stuttgart.

15. Lenfant, C. (1974) in: Carbon dioxide and metabolic regulations (Nahas, G. \& S Schäfer, K. E., eds.) Springer New YorkHeidelberg-Berlin.

16. Siggaard-Andersen, O. (1963), J. Clin. Lab. Invest. 15 , 211-217.

17. Kelman, G. R. \& Nunn, J. F. (1966), J. Appl. Physiol. 21, 1484-1490.
Dr. O. Müller-Plathe Zentrallaboratorium Allg. Krankenhaus Altona Paul-Ehrlich-Straßa 1 2000 Hamburg 50 